\title{
STEAP1 Positive
}

National Cancer Institute

\section{Source}

National Cancer Institute. STEAP1 Positive. NCI Thesaurus. Code C139793.

Indicates that STEAP1 expression has been detected in a sample. 